Proceedings of the 1999 Particle Accelerator Conference, New York, 1999

\title{
BEAM-BEAM EFFECT AND DYNAMIC APERTURE OF THE MUON COLLIDER*
}

\author{
M. A. Furman ${ }^{\dagger}$, Center for Beam Physics, LBNL, Berkeley, CA, USA
}

\section{Abstract}

We consider the combined effects of the beam-beam interaction and the lattice nonlinearities for one specific design of the muon collider ring. The beam-beam interaction is represented by a thick gaussian lens, and the ring is described by a 9th-order Taylor map. At the single-particle level we compare the tune footprints and the dynamic aperture with and without the beam-beam effect. At the multiparticle level, using a "weak-strong" description of the beam-beam interaction, we compare the time evolution of the rms beam sizes with a linear lattice map and with the full nonlinear map.

\section{INTRODUCTION}

The desire to have a muon collider with high luminosity combined with the instability of the muon places serious constraints on the lattice design of this machine. In particular, lattice nonlinearities are severe, and the beam-beam interaction has a strength comparable to a mature $e^{+} e^{-}$ collider [1]. Traditionally, the beam-beam interaction and lattice dynamics have been studied separately. It is likely that the muon collider will be the first machine in which these two pieces of dynamics, along with wakefield effects, may have to be studied in combination. In this note we present an initial step in this direction, improving upon a previous study [2] with linear lattices, by carrying out single-particle and multiparticle tracking calculations for one specific design of the muon collider whose relevant parameters are listed in Table 1. We represent the lattice by a 9th-order Taylor map obtained from the program COSY INFINITY [3], and the beam-beam interaction by a thick lens. We present our results in three cases: (a) linear part of the map plus beam-beam, (b) full 9th-order map without beam-beam, and (c) full 9th-order map plus beam-beam. We compute beam footprints, dynamic aperture plots and time evolution of the rms beam sizes.

There are several limitations in this investigation which we expect to improve upon in the future: (1) the beambeam interaction is represented only in weak-strong mode, albeit in thick lens approximation (i.e., nonzero bunch length effects included) assuming a tri-gaussian density distribution, (2) the longitudinal beam-beam forces are wholly neglected, and (3) the 9th-order map does not incorporate RF cavities.

\footnotetext{
${ }^{*}$ Work supported by the US Department of Energy under contract no. DE-AC03-76SF00098.

${ }^{\dagger}$ E-mail address: mafurman@1bl.gov
}

Table 1: Selected muon collider parameters.

\begin{tabular}{lr}
\hline \hline Circumference, $C[\mathrm{~m}]$ & 345 \\
Beam energy, $E[\mathrm{GeV}]$ & 50 \\
Relativistic factor, $\gamma$ & 473.2 \\
No. of particles per bunch, $N$ & $4 \times 10^{12}$ \\
Beta function at the IP, $\beta^{*}[\mathrm{~cm}]$ & 4 \\
Normalized emittance, $\epsilon_{N}[\mu \mathrm{m}-\mathrm{rad}]$ & 85 \\
RMS beam size at the IP, $\sigma_{0}[\mu \mathrm{m}]$ & 84.76 \\
RMS bunch length, $\sigma_{z}[\mathrm{~cm}]$ & 4 \\
Relative momentum spread, $\sigma_{p} / p$ & $1.2 \times 10^{-4}$ \\
Nominal beam-beam parameter, $\xi$ & 0.051 \\
Tunes, $\nu_{x} / \nu_{y}$ & $0.862 / 0.639$ \\
\hline \hline
\end{tabular}

\section{CALCULATION}

In Table 1, the four beta functions at the interaction point (IP) are equal, as are the four emittances. The beam-beam element consists of a static trigaussian thick lens implemented by dividing the bunch into 5 kicks whose weights and longitudinal positions follow the "equal-weight" prescription [4]. The beams collide head-on at the IP. The longitudinal beam-beam force is wholly neglected. The transverse force from each of the 5 kicks is computed from the well-known expression of the electric field of a round gaussian beam. A particle from the dynamical beam is consecutively kicked by each of these 5 kicks; in between successive kicks, the particle undergoes simple drifts.

The lattice is represented by a 9th-order Taylor expansion of the 6-dimensional one-turn map at the IP, obtained from COSY INFINITY [3]. It does not include RF cavities. The beta functions and tunes listed in Table 1 are extracted from the linear part of the map, which also has $\beta^{\prime}=0$ at the IP. The action of the map on each particle of the dynamical beam is expressed by the polynomial

$$
\begin{aligned}
z_{i}^{\prime}=\sum_{\ell=0}^{L} \sum_{\left\{n_{k}\right\}_{\ell}} C_{i}^{(\ell)}\left(n_{1}, n_{2}, n_{3}, n_{4}, n_{5}, n_{6}\right) \\
\quad \times z_{1}^{n_{1}} z_{2}^{n_{2}} z_{3}^{n_{3}} z_{4}^{n_{4}} z_{5}^{n_{5}} z_{6}^{n_{6}}
\end{aligned}
$$

where $z_{i}$, with $i=1, \cdots, 6$, represents the (suitably defined [3]) $i$-th coordinate of the particle at turn $t, z_{i}^{\prime}$ is the coordinate at turn $t+1, \ell$ is the order of the expansion, with maximum order $L=9$, and $\sum_{\left\{n_{k}\right\}_{\ell}}(\cdots)$ stands for a summation over all possible combinations of the $n_{k}$ 's such that $\sum_{k} n_{k}=\ell$ with $n_{k} \geq 0$. The map is described by the full set of $C_{i}^{(\ell)}$ 's; in the particular case we study, there are 5040 nonzero such coefficients. Eq. 1 is applied to every 
particle in the dynamical beam.

In single-particle mode we track one particle in the dynamical beam with given initial coordinates $x_{0}$ and $y_{0}$. By successively letting $x_{0}$ and $y_{0}$ take on values in a rectangular grid we can generate a tune footprint. We extract the tunes by numerically integrating the phases of the particle over 500 turns. To determine the dynamic aperture, we track the particle for 1000 turns. In all cases the initial values of the remaining coordinates $\left(x^{\prime}, y^{\prime}, z\right.$ and $\left.\Delta p\right)$ are set to 0 . In order to avoid numerical overflow problems, we specify the criterion that a particle is outside the dynamic aperture if at any turn it is outside a sphere in $x, y, z$ about the bunch center with radius $1 \mathrm{~m}$.

In multiparticle mode we start from an initial 6D gaussian beam distribution of particles centered at the origin with the $\sigma$ 's specified in Table 1 or extracted from these parameters. In all cases we choose 10,000 representative particles. We then track this distribution for 1000 turns and compute and record the rms values at every turn.

In all cases we take the muon to be a stable particle. There are no physical apertures in the calculation. Radiation and quantum excitation are ignored, as are the mutual interactions of the particles within the bunch.

\section{RESULTS}

\subsection{Footprints}

Footprints are shown in Fig. 1. We tracked 100 particles whose initial conditions were on a $10 \times 10$ grid with $x_{0} / \sigma, y_{0} / \sigma=0.1,1,1.5,2,2.5,3,3.5,4,4.5,5$. It is seen that resonances do not cause any distortion of the footprint in the case of linear map + beam-beam. There are severe distortions for the case of full map with or without beam-beam presumably due to island trapping.

\subsection{Dynamic aperture}

Figure 2 shows the dynamic aperture plots for the case of nonlinear map with and without beam-beam (the dynamic aperture for the case of linear map + beam-beam is obviously infinite). It can be seen that the beam-beam interaction has a slight effect on dynamic aperture.

\subsection{Beam size evolution}

Figure 3 shows the time evolution over 1000 turns of the normalized beam sizes, $\sigma_{x} / \sigma_{0}$ and $\sigma_{y} / \sigma_{0}$. It is seen that the cases with beam-beam, with a linear map or with a full map, are very similar to each other. This implies that the map has little effect on beam-beam performance. Even though the beam starts out with $\sigma_{x}=\sigma_{y}$, the dynamical beam sizes quickly become different. This is due to the dynamical beta function effect [5] which implies $\sigma_{x} \neq \sigma_{y}$ whenever $\nu_{x} \neq \nu_{y}$.

In the absence of the beam-beam element, the beam sizes are also unequal, but in this case the difference is due purely to a " $\sqrt{N}$ " fluctuation of the initial particle distribution.
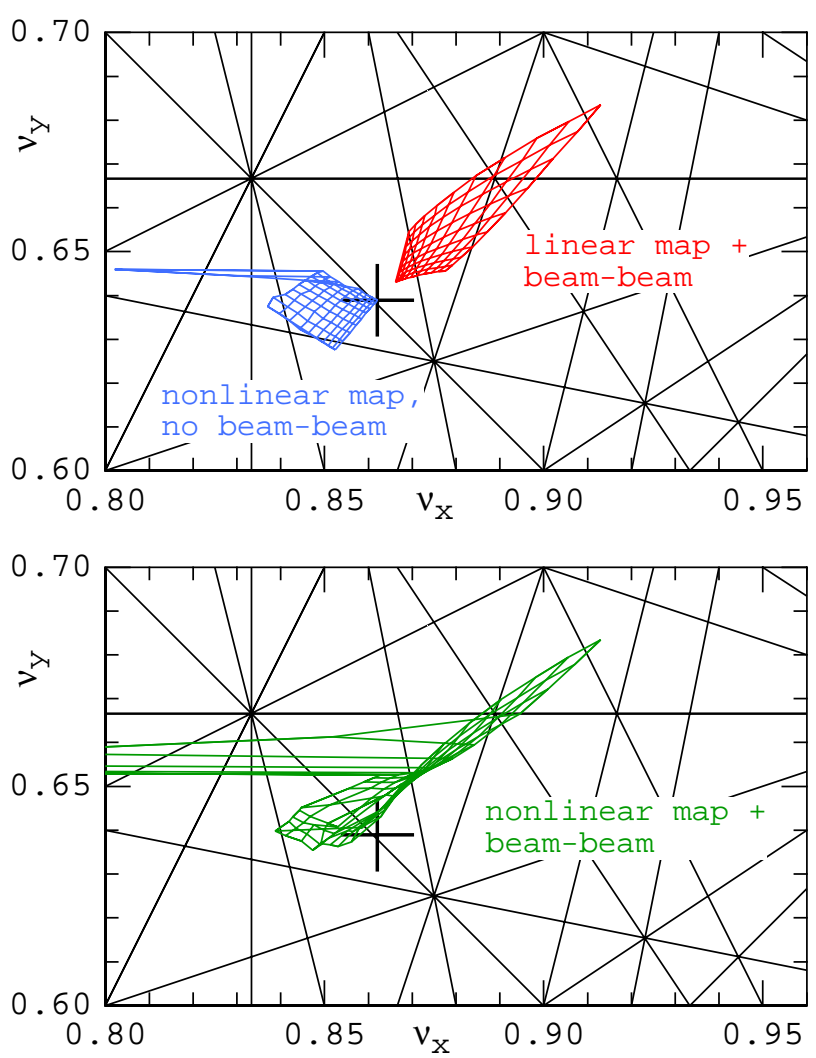

Figure 1: Tune footprints of the beam. Top: linear map + beam-beam and nonlinear map without beam-beam. Bottom: nonlinear map + beam-beam. The cross is at the working point defined by the map. Resonance lines up to 6-th order are shown.

\section{DISCUSSION}

The results presented here are to be taken only as an initial step in a more systematical examination of beam dynamics in the muon collider. It is desirable to study maps that better describe the machine, including RF cavities. It is also desirable to improve the beam-beam element so that it includes longitudinal forces, which have recently shown to be important when the momentum spread of the beam is very small [6].

Although preliminary, the present results confirm the generally accepted belief, derived from theory and experiment with $e^{+} e^{-}$colliders, that lattice nonlinearities do not affect the beam-beam dynamics significantly near the beam core. It is gratifying that this is the case even in this design, whose dynamic aperture is markedly smaller than in typical $e^{+} e^{-}$machines. We conclude that the luminosity performance will not be detrimentally affected by nonlinearities. We expect this conclusion to remain valid as long as the dynamic aperture is at least a few $\sigma$ 's.

On the other hand, we expect that nonlinearities will have a significant effect on the large-amplitude tails of the beam, whose dynamics is quite different from the beam core. This issue remains to be studied. 

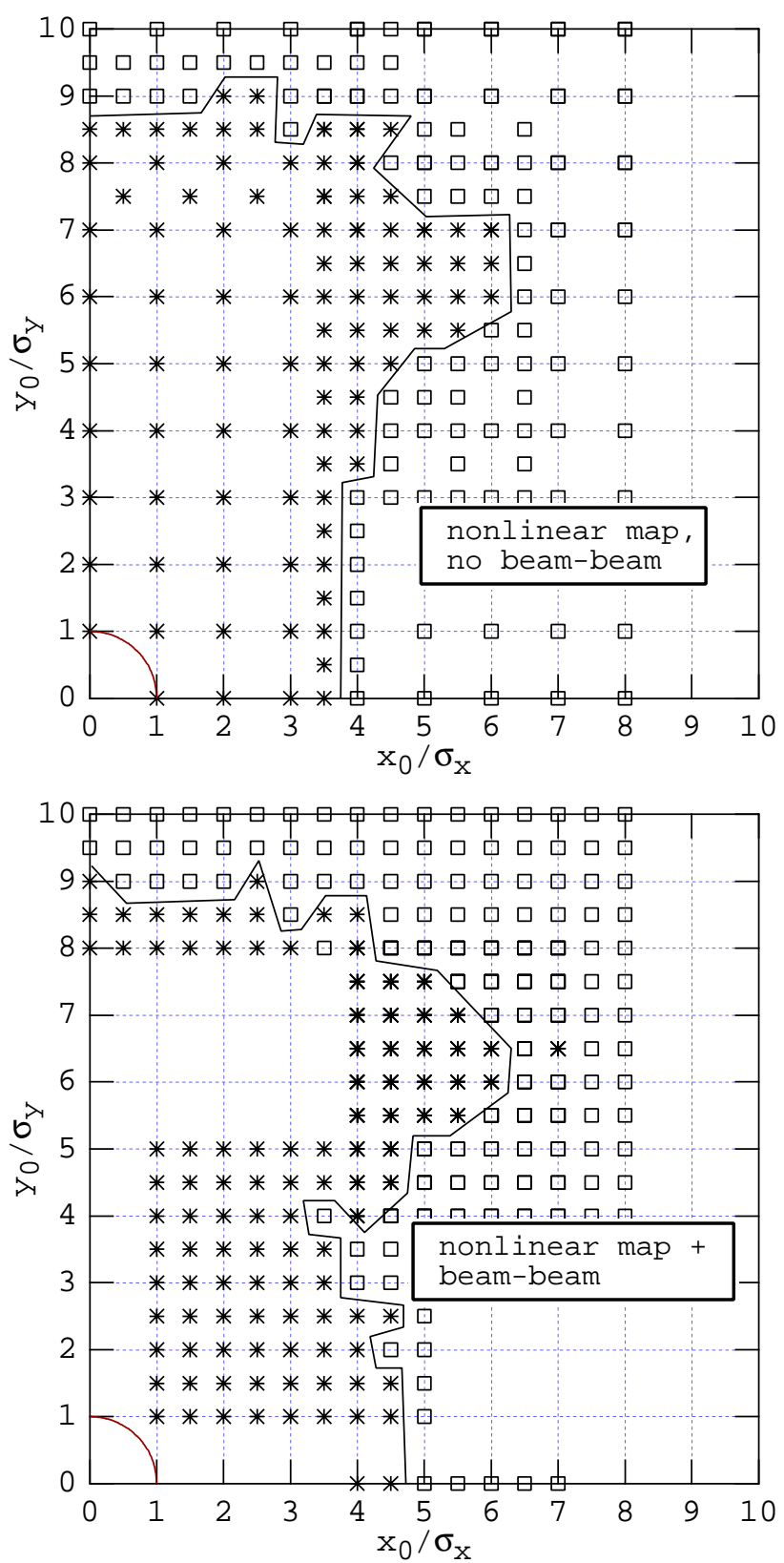

Figure 2: Top: dynamic aperture for the map without beambeam. Bottom: map + beam-beam. Crosses indicate particles that survived for 1000 turns, boxes those that did not. The hand-drawn polygon is meant to guide the eye along the dynamic aperture boundary. The quarter-circle at the lower left corner is the $1-\sigma$ beam profile.

\section{ACKNOWLEDGMENTS}

I am grateful to C. Johnstone and W. S. Wan for providing me with a COSY INFINITY map, to R. Palmer for discussions and clarification of machine parameters, and to NERSC for supercomputer support.
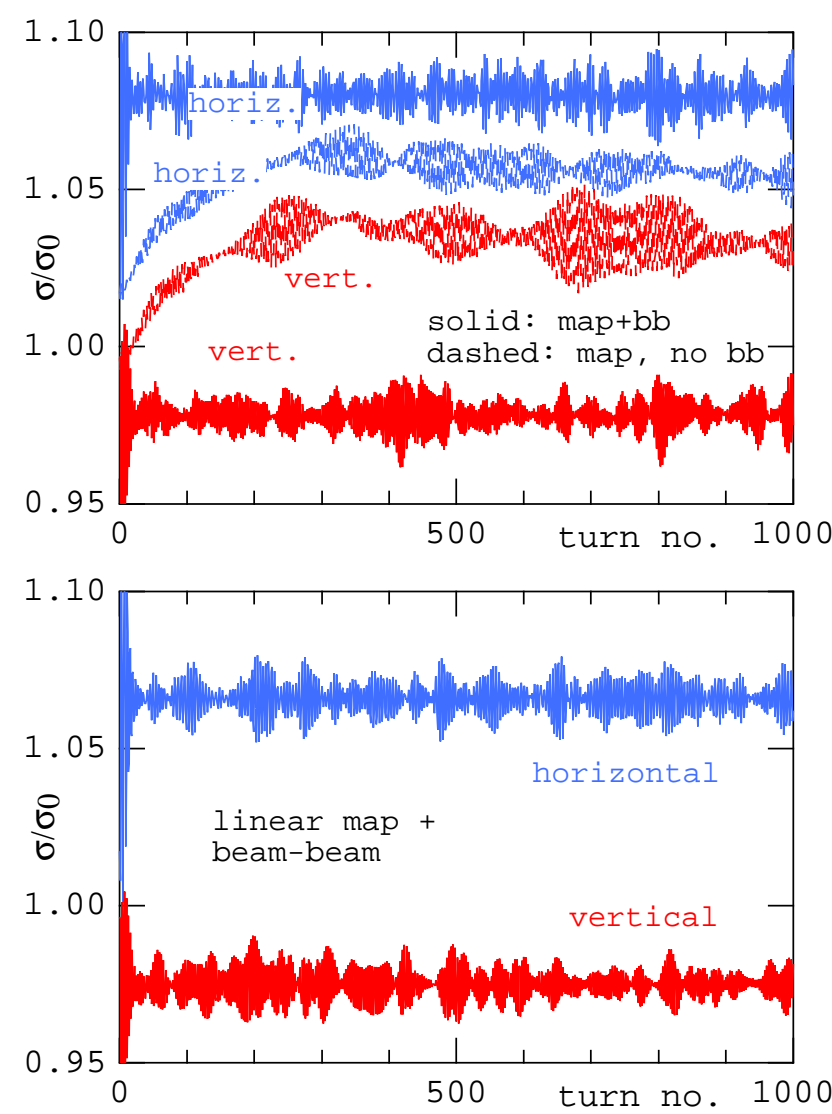

Figure 3: RMS beam size evolution. Top: nonlinear map with and without beam-beam. Bottom: linear map + beambeam.

\section{REFERENCES}

[1] "Status of Muon Collider R\&D and Future Plans," BNL-65623/Fermilab-PUB-98/179/LBNL-41935, Sept. 1998.

[2] M. A. Furman, "The Classical Beam-Beam Interaction for the Muon Collider: A First Look," $\mu^{+} \mu^{-} \mathrm{Col}$ lider: A Feasibility Study, BNL-52503/Fermi Lab-Conf-96092/LBNL-38946, July 1996, sec. 8.6; Proc. 1996 Snowmass Workshop New Directions for High-Energy Physics; http://www.lbl.gov/ miguel/mucoll_snowmass96.pdf.

[3] M. Berz, "COSY INFINITY v8 User's Guide and Reference Manual," Nov. 7, 1997.

[4] M. A. Furman, A. A. Zholents, T. Chen and D. Shatilov, "Comparisons of Beam-Beam Simulations," Proc. Seventh Advanced ICFA Workshop on Beam Dynamics, JINR, Dubna, Russia, 18-20 May, 1995, pp. 123-142; http://www.lbl.gov/ miguel/BBcompICFA.pdf.

[5] M. A. Furman, "Beam-Beam Tune Shift and Dynamical Beta Function in PEP-II," Proc. EPAC94, London, England, June 27-July 1, 1994, p. 1145, http://www.lbl.gov/ miguel/epac94_dynbeta_LBNLrep.pdf.

[6] E. S. Kim, A. M. Sessler and J. Wurtele, "Control of energy spread due to the beam-beam interaction in a $50 \mathrm{GeV} \times 50$ $\mathrm{GeV}$ muon collider ring," in preparation. 